\title{
Correlation between apparent diffusion coefficient and viscoelasticity of articular cartilage in a porcine model
}

\author{
T. Aoki $\cdot$ A. Watanabe $\cdot$ N. Nitta $\cdot$ T. Numano $•$ \\ M. Fukushi • M. Niitsu
}

Received: 12 September 2011 /Revised: 25 November 2011 /Accepted: 28 November 2011 /Published online: 11 January 2012

(C) The Author(s) 2012. This article is published with open access at Springerlink.com

\begin{abstract}
Objective Quantitative MR imaging techniques of degenerative cartilage have been reported as useful indicators of degenerative changes in cartilage extracellular matrix, which consists of proteoglycans, collagen, non-collagenous proteins, and water. Apparent diffusion coefficient (ADC) mapping of cartilage has been shown to correlate mainly with the water content of the cartilage. As the water content of the cartilage in turn correlates with its viscoelasticity, which directly affects the mechanical strength of articular cartilage, ADC can serve as a potentially useful indicator of the mechanical strength of cartilage. The aim of this study was to investigate the correlation between $\mathrm{ADC}$ and viscoelasticity as measured by indentation testing.

Materials and methods Fresh porcine knee joints $(n=20$, age 6 months) were obtained from a local abattoir. ADC of porcine knee cartilage was measured using a 3-Tesla
\end{abstract}

T. Aoki $(\bowtie)$

Department of Radiological Science, Graduate School of Human

Health Science, Tokyo Metropolitan University,

7-2-10 Higashiogu,

Arakawa-ku, Tokyo, Japan

e-mail: aoki-takako@hs.tmu.ac.jp

\section{A. Watanabe}

Department of Orthopedic Surgery,

Teikyo University Chiba Medical Center,

3426-3 Anesaki,

Ichihara, Chiba 299-0111, Japan

e-mail: atsuyan1@pa2.so-net.ne.jp

\section{N. Nitta}

Human Technology Research Institute, National Institute of

Advanced Industrial Science and Technology (AIST),

1-2-1 Namiki,

Tsukuba, Ibaraki, Japan

e-mail: n.nitta@aist.go.jp
MRI. Indentation testing was performed on an electromechanical precision-controlled system, and viscosity coefficient and relaxation time were measured as additional indicators of the viscoelasticity of cartilage. The relationship between $\mathrm{ADC}$ and viscosity coefficient as well as that between $\mathrm{ADC}$ and relaxation time were assessed.

Results $\mathrm{ADC}$ was correlated with relaxation time and viscosity coefficient $\left(\mathrm{R}^{2}=0.75\right.$ and 0.69 , respectively, $\left.p<0.01\right)$. The mean relaxation time values in the weight-bearing and nonweight-bearing regions were $0.61 \pm 0.17 \mathrm{~ms}$ and $0.14 \pm 0.08 \mathrm{~ms}$, respectively.

Conclusions This study found a moderate correlation between ADC and viscoelasticity in the superficial articular cartilage. Both molecular diffusion and viscoelasticity were higher in weight bearing than non-weight-bearing articular cartilage areas.

T. Numano $\cdot$ M. Fukushi

Faculty of Health Sciences School of Radiological Science,

Tokyo Metropolitan University,

7-2-10 Higashiogu,

Arakawa-ku, Tokyo, Japan

T. Numano

e-mail: t-numano@hs.tmu.ac.jp

M. Fukushi

e-mail: fukushi@hs.tmu.ac.jp

M. Niitsu

Department of Radiology, Saitama Medical University Hospital, 38 Morohongo, Moroyama-cho, Iruma-gun, Saitama, Japan

e-mail:niitsu@saitama-med.ac.jp 
Keywords Apparent diffusion coefficient . Viscoelasticity . Articular cartilage · Water content · Quantitative MR imaging

\section{Introduction}

In the early stages of $\mathrm{OA}$, conservative treatment is often recommended to prevent the progression of degenerative changes in articular cartilage. Yet the early degenerative changes in articular cartilage associated with OA are often invisible in images acquired through roentgenography, and conventional MR imaging. These changes include the disruption and/or alteration of the extracellular matrix, in such forms as decreased concentration of proteoglycans, increased water content, or deterioration of collagenous architecture, all of which have been demonstrated biologically and histologically $[1,2]$.

The application of quantitative MR imaging techniques to degenerative cartilage has been a focus of recent research $[3,4]$. Quantitative MR imaging techniques, such as T2 relaxation time mapping $[5,6]$, T1 $\rho$ relaxation time mapping [7-9], delayed gadolinium-diethylene-triamine-penta-acetic (Gd-DTPA)-enhanced MR imaging of cartilage (dGEMRIC) [10], and apparent diffusion coefficient (ADC) mapping [11], have been reported as useful indicators of degenerative changes in cartilage extracellular matrix, which consists of proteoglycans, collagen, non-collagenous proteins, and water. T2 measurements in cartilage have been shown to correlate with collagenous architecture and water content [5], while $\mathrm{T} 1 \rho$ measurements in cartilage have been shown to correlate with proteoglycans and water content [12]. The dGEMRIC technique has been shown to correlate mainly with proteoglycans.

ADC measurements, on the other hand, have been shown to correlate mainly with water content and collagen matrix structure in cartilage $[13,14]$. It has been shown that water and the collagen matrix produces the flow-dependent viscoelasticity of cartilage [15]. Thus, ADC can serve as an ideal indicator of the viscoelasticity of cartilage.

Several studies have addressed the relationship between $\mathrm{ADC}$ and viscoelasticity. Juras et al. reported that ADC correlates with relaxation time in superficial zones using specimens in different stages of degeneration from patients who had undergone total knee replacement surgery [16]. Topographic variation in the cartilage matrix has also been shown to exist [17-19]: weight-bearing regions receive direct compression and tensile loading during walking, while non-weight-bearing regions receive less loading. Yet, topographic variation in terms of ADC and viscoelasticity has not been previously studied. The aim of this study was to investigate the correlation between ADC and viscoelasticity using porcine knee cartilage, especially focusing on the difference between weight-bearing and non-weight-bearing region.

\section{Methods}

Subjects

Fresh porcine knee joints ( $n=20$, age 6 months) were obtained from a local abattoir (ZEN-NOH Central Research Institute for Feed and Livestock, Ibaraki, Japan). Because we used branded edible pigs, which for quality-control purposes are slaughtered precisely 6 months after birth, all pigs had almost finished growing but remained skeletally immature.

The study was approved by the institutional review board.

\section{MR imaging}

MR imaging was performed within $30 \mathrm{~h}$ after euthanasia. Specimens were kept at room temperature $\left(20^{\circ} \mathrm{C}\right)$ for $3 \mathrm{~h}$ before MR imaging. MR imaging was performed on a 3.0-T whole-body clinical scanner (Intera Achieva; Philips Medical Systems, Best, Netherlands) with an 8-ch SENSE (sensitivity encoding) knee coil using a parallel imaging technique. Sagittal MR images were acquired along a plane perpendicular to the line that passes though the medial femoral condyle and the lateral femoral condyle. In this plane, we did not evaluate the tibial and patellar cartilage to avoid any partial volume effect due to surrounding structures. Morphological isotropic images were acquired using a three-dimensional (3D)-fast field echo (FFE) sequence with the following parameters: repetition time (TR)/echo time (TE1/TE2) 19/7.0/13.3 ms, field of view (FOV) $150 \times 150 \mathrm{~mm}$, interpolated matrix $512 \times 512$ (acquired matrix $256 \times 256$ ), slice thickness $0.3 \mathrm{~mm}$, and number of excitations (NEX) 1 (total scan duration 5 min 09 s). ADC of cartilage was measured using a single-shot spin echo-echo planar image sequence with the following parameters: TR/TE $4,000 / 47 \mathrm{~ms}$, FOV $120 \times 120 \mathrm{~mm}$, interpolated matrix $256 \times$ 256 (acquired matrix $128 \times 128$ ), slice thickness $3 \mathrm{~mm}$, gap $0.3 \mathrm{~mm}$, number of slices 19, and NEX 1 (total scan duration $1 \mathrm{~min} 48 \mathrm{~s}$ ). Diffusion gradients were applied with $b$ values of $0,700,1,000$, and $1,500 \mathrm{~s} / \mathrm{mm}^{2}$. An ADC map was generated from diffusion-weighted images using the built-in software that accompanies the clinical scanner (Philips). An ADC map was generated on a pixel-by-pixel basis by fitting the $b$ value data from the measured signal intensity $\left(S_{b}\right)$ attenuation according to a mono-exponential decay equation, as follows:

$S_{(b)}=S_{(b=0)} \exp (-b D)$

\section{ROI setting}

Four sites in each specimen, namely the medial femoral condyle, the lateral femoral condyle, the medial trochlea, and the lateral trochlea, were analyzed by means of both MR 
imaging and indentation testing (Figs. 1, 2). For each specimen, a region of interest (ROI) was drawn on the slice that passed through the center of the medial femoral condyle, the lateral femoral condyle, the medial trochlea, and the lateral trochlea. This ROI was drawn to include the weight-bearing area of the medial and lateral condyles and the non-weightbearing area of the medial and lateral trochlea. Furthermore, the cartilage was divided along a line parallel to the cartilage-bone interface into two layers (superficial and deep) of equal thickness. An ROI was drawn over the entire superficial layer as it has been shown that degenerative changes begin in the superficial layer, and also because mechanical testing mainly reflects the properties of the superficial layer of cartilage. All ROIs were drawn manually by a single investigator.

\section{Mechanical testing}

Indentation testing was performed on an electromechanical precision-controlled system (Vesmeter E-200DT; WaveCyber Co., Ltd., Saitama, Japan). The shape of the indenter tip is a cone with an angle of $30^{\circ}$, a tip diameter of $0.1 \mathrm{~mm}$, and a pressurization of $35 \mathrm{~g}$ each $0.2 \mathrm{~s}$. Measuring one region takes less than $2 \mathrm{~min}$.

This device can provide the viscosity, elasticity, relaxation time, elastic rate, stiffness, and strain depth as given by Voigt's equation:

$S=G \gamma+\eta \cdot \frac{d \gamma}{d t}$

where $\gamma$ denotes displacement, $G$ is elastic modulus, $\eta$ denotes viscosity, and $S$ is stress. Indentation testing was performed on the same regions that were selected for MRI analysis. Two lines consisting of five points per line at intervals of 3-4 mm were tested for a total of ten points in each region. Viscosity coefficient and relaxation time were measured as indicators of the viscoelasticity of cartilage. To minimize the possibility of measurement errors during indentation tests, the mean viscosity coefficient and relaxation time obtained for all ten points in each region were taken as the viscosity coefficient and relaxation time for that region. During mechanical testing, the specimens were wrapped in moist gauze to prevent them from drying.

\section{Statistical analysis}

The relationship between ADC and viscosity coefficient as well as that between ADC and relaxation time were assessed by means of correlation analysis. The correlation coefficients were assessed using a Pearson coefficient. Significant differences among the weight-bearing, non-weight-bearing, medial, and lateral regions were evaluated by multiple comparison tests using one-way analysis of variance (ANOVA). Statistical significance was defined as $p<0.05$. Statistical software (Statcel for Windows, OMS, Saitama, Japan) was used for all analyses.

\section{Results}

All porcine knees were visually healthy, with no blistering, ulceration, fissuring, or thinning of cartilage. ADC was correlated with relaxation time and viscosity coefficient
Fig. 1 Indentation testing device (a) and the appearance under measurement of porcine knee cartilage in situ (b). A shape of the tip of the indenter is cone of $30^{\circ}$, tip diameter of $0.1 \mathrm{~mm}$, and pressurization of $35 \mathrm{~g}$

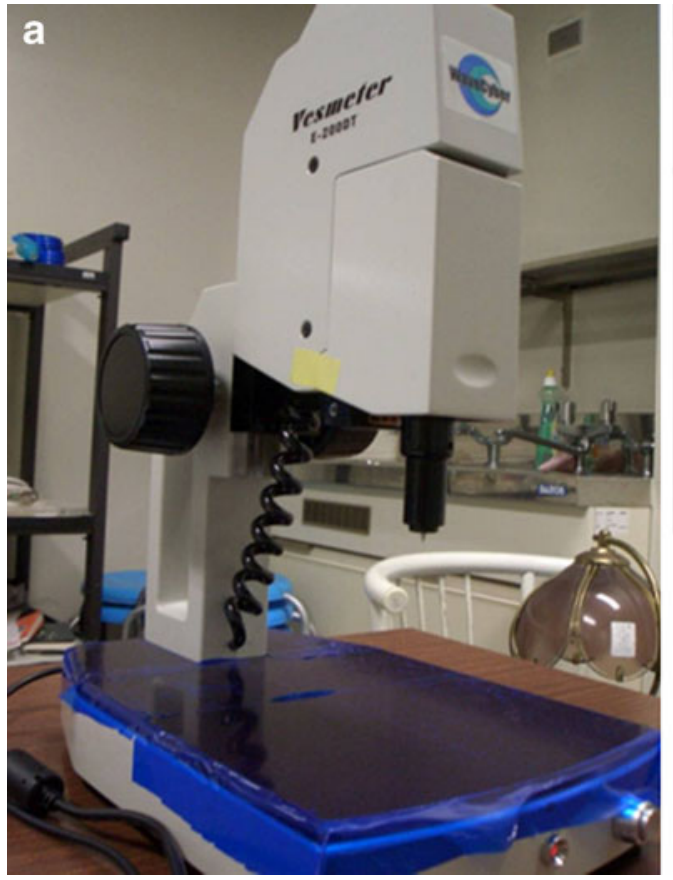


Fig. 2 MR image of weightbearing region (a) and nonweight-bearing region (b), indenter testing points of weightbearing region (c) and nonweight-bearing region $(\mathbf{d})$
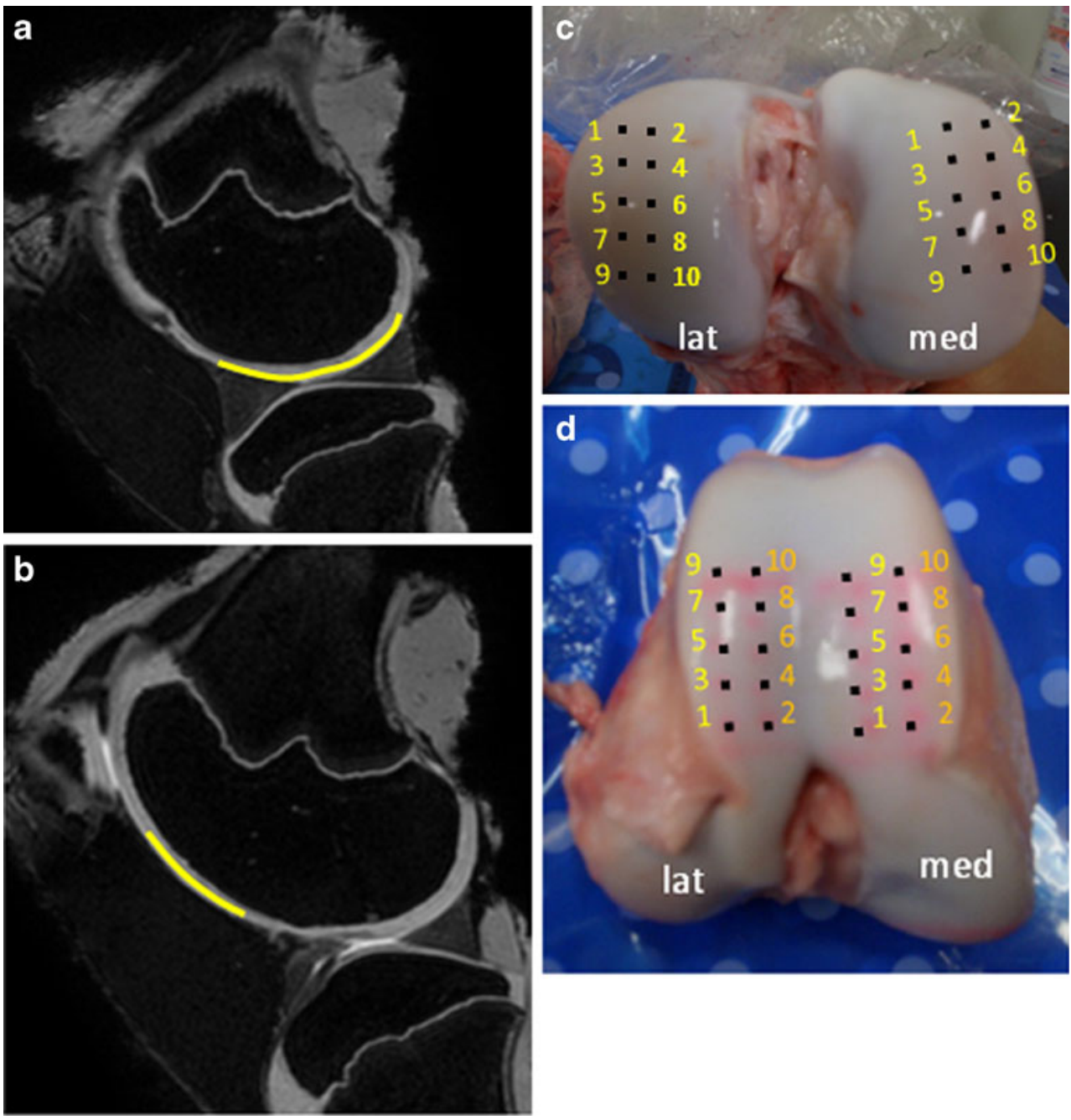

$\left(\mathrm{R}^{2}=0.75\right.$ and 0.69 , respectively, $\left.p<0.01\right)$ (Figs. 3,4$)$. The mean relaxation time values in the weight-bearing and nonweight-bearing regions were $0.61 \pm 0.17 \mathrm{~ms}$ and $0.14 \pm$ $0.08 \mathrm{~ms}$, respectively (Table 1 ). The mean viscosity

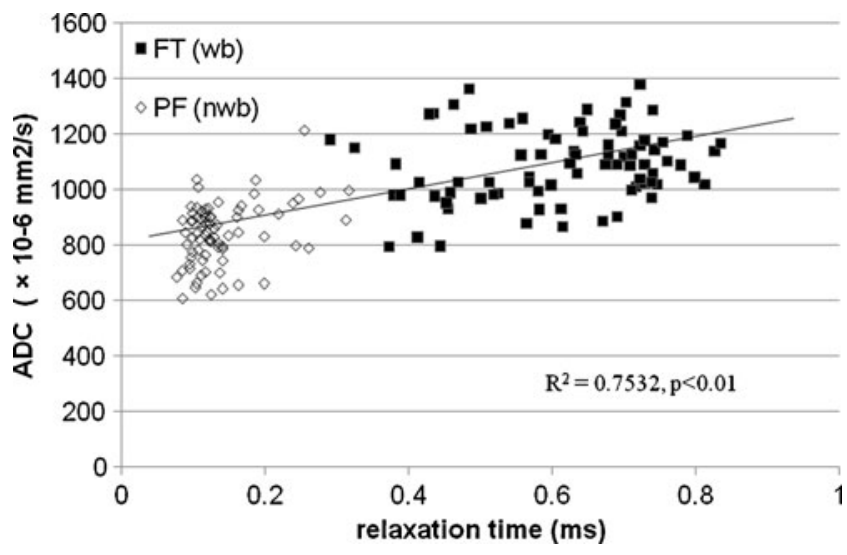

Fig. 3 Correlation between $\mathrm{ADC}$ and relaxation time. A significant correlation between ADC and relaxation time was observed. The ADC of the weight-bearing region (FT) is significantly higher than that of the non-weight-bearing region $(\mathrm{PF})$ coefficient values in weight-bearing and non-weight-bearing regions were $5,043 \pm 787 \mathrm{kPa}$ s and $3,100 \pm 806 \mathrm{kPa} \mathrm{s}$, respectively (Table 1). Weight-bearing regions had significantly longer relaxation times and higher viscosity coefficient values

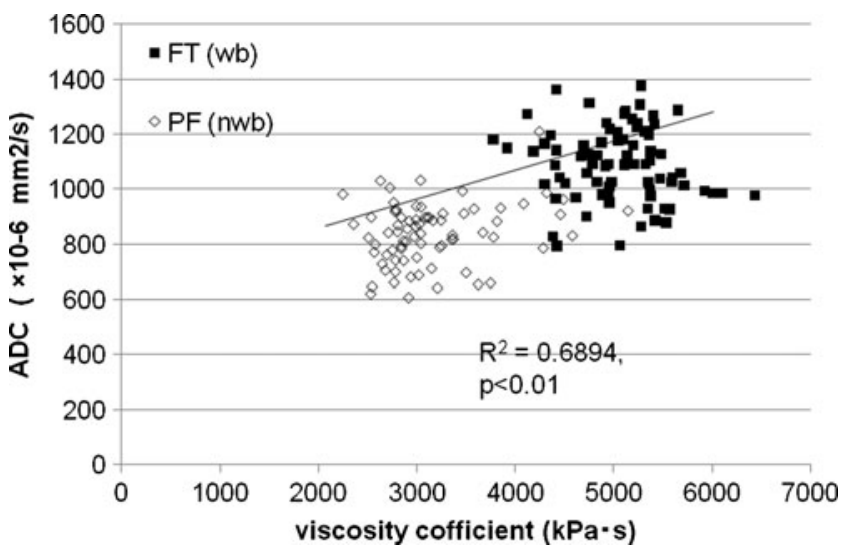

Fig. 4 Correlation between ADC and viscosity coefficient. A significant correlation between ADC and viscosity coefficient was observed. The ADC and the viscosity coefficient in the weight-bearing region is significantly higher than that of the non-weight-bearing region 
Table 1 ADC and mechanical property of cartilage

\begin{tabular}{lll}
\hline & $\begin{array}{l}\text { Weight-bearing } \\
\text { region }\end{array}$ & $\begin{array}{l}\text { Non-weight-bearing } \\
\text { region }\end{array}$ \\
\hline $\mathrm{ADC}\left(\mathrm{x} 10^{-6} \mathrm{~mm}^{2} / \mathrm{s}\right)$ & $1,088 \pm 125^{*}$ & $835 \pm 110^{*}$ \\
$\begin{array}{c}\text { Viscosity coefficient } \\
\left(\mathrm{N} \cdot \mathrm{s} / \mathrm{m}^{2}\right)\end{array}$ & $5,043 \pm 787^{*}$ & $3,100 \pm 806^{*}$ \\
Relaxation time $(\mathrm{ms})$ & $0.61 \pm 0.17^{*}$ & $0.14 \pm 0.08^{*}$ \\
\hline
\end{tabular}

${ }^{*} p<0.05$

than non-weight-bearing regions did $(p<0.05)$. The mean $\mathrm{ADC}$ values in weight-bearing and non-weight-bearing regions were $1,087 \pm 125 \times 10^{-6} \mathrm{~mm}^{2} / \mathrm{s}$ and $835 \pm 110 \times 10^{-6} \mathrm{~mm}^{2} / \mathrm{s}$, respectively (Table 1). All of these differences were statistically significant $(p<0.05)$. Figure 5 shows sagittal images of weightbearing and non-weight-bearing regions with ADC mapping.

\section{Discussion}

Cartilage plays a critical role in joint function, where it acts as a shock absorber during joint loading. This function is partly enabled by proteoglycan, which has a high negative charge and binds to water molecules, thereby generating the swelling pressure of cartilage [20]. This swelling pressure is counteracted by the tensile strength of cartilage, which is provided by a dense and regularly arranged collagen network [21-24]. Therefore, intermolecular interactions of both major components of cartilage with water molecules give cartilage its viscoelasticity, the characteristic that enables cartilage to act as a shock absorber $[15,25]$. The decrease in proteoglycans and disruption of the collagen network that occurs over time in degenerative cartilage leads to a loss of viscoelasticity [16].

It has been suggested that differences in diffusivity as assessed by ADC may be due to differences in water mobility, which in turn is determined by the average pore size in the cartilage matrix $[26,27]$. The difference in diffusivity between tibiofemoral and patellofemoral cartilage, for example, might be due to differences in their inherent matrix structures and compositions. Indentation testing can directly evaluate the viscoelasticity of cartilage, and has been used in several studies to assess cartilage function in degenerative cartilage [28]. Recent studies have used arthroscopic indentation instruments for this purpose, but such indentation testing is somewhat invasive and should not be used as a routine clinical evaluation method. In comparison, MR imaging is less invasive and can be used more regularly for clinical evaluation. This study has uncovered significant correlations between ADC and relaxation time, as well as between ADC and viscosity coefficient, indicating that $\mathrm{ADC}$ can serve as an ideal measure of cartilage viscoelasticity.

There are several limitations of this study. First, only visually healthy cartilage specimens were recruited. Thus, the usefulness of ADC as a means of assessing degenerative cartilage remains unknown. In addition, as biochemical and histological analysis were not performed, the usefulness of $\mathrm{ADC}$ as a quantitative measure of cartilage degeneration is also unknown. These deficiencies constitute major weaknesses of this study.

As cartilage samples from several different regions of several different porcine knees were used, specimens with various cartilage matrix compositions were included. Therefore, the significant correlation observed between ADC and viscoelasticity lends support to the notion that ADC could be useful in the evaluation of degenerative cartilage as well. Further studies including knees with degenerative cartilage and incorporating biochemical and histological analysis are needed to confirm this. At present, however, it is unknown whether the results of the current study may be applied to the evaluation of repaired cartilage, degenerating cartilage, and/or mature knees.

A second limitation is that the indentation test was the only mechanical test performed on our cartilage samples. It has been shown that cartilage exhibits a much greater stiffness in tension than in compression, and that cartilage
Fig. 5 ADC mapping with distortion correction of a weight-bearing region (a) and a non-weight-bearing region (b)
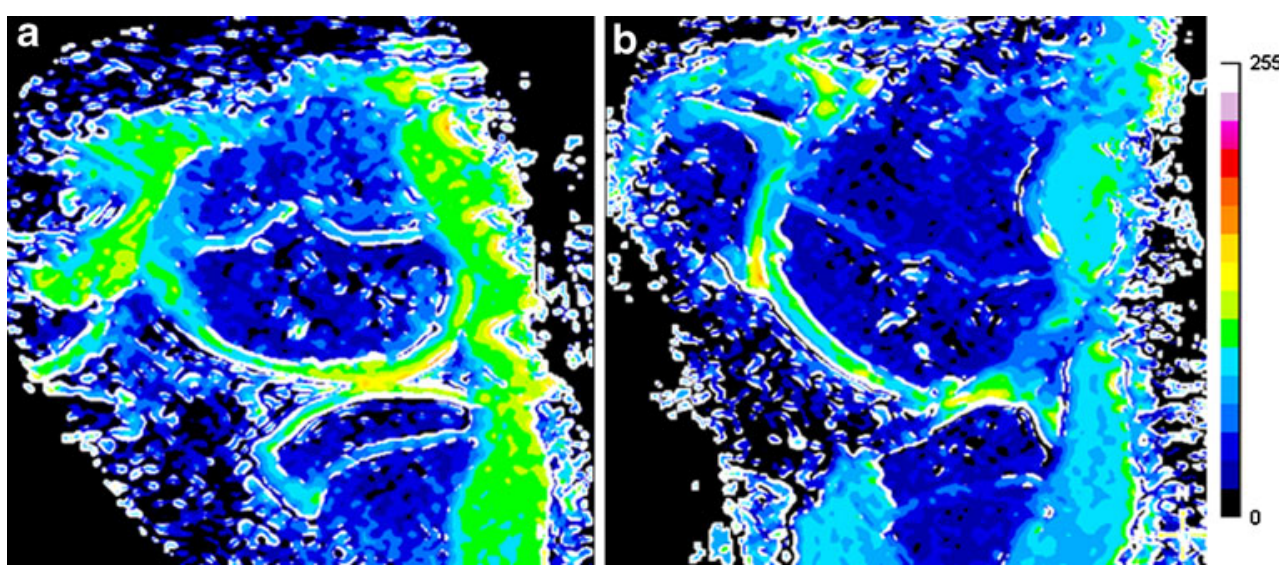
exhibits anisotropy in tension and compression. Further studies using other mechanical tests including tension tests may reveal a more detailed relationship between ADC and data on cartilage strength.

Third, we did not use any models of defective or degenerating cartilage in this study, as we used only tissues from branded edible pigs, and as only immature porcine knees were included. It will be necessary to determine whether similar results can be obtained using repaired cartilage, degenerating cartilage, and/or mature knee cartilage in future studies before ADC is used clinically as a measure of cartilage viscoelasticity.

In conclusion, a moderate correlation was observed between ADC and viscoelasticity in superficial articular cartilage. Both molecular diffusion and viscoelasticity were higher in weight-bearing than in non-weight-bearing regions of articular cartilage.

Acknowledgments The authors would like to acknowledge the help and advice of Atsuto Hoshikawa, who provided insightful comments and suggestions, and N. Ochiai, a researcher of porcine knee joints, who provided fresh specimens from ZEN-NOH.

Conflict of Interest My colleagues and I certify that we have no financial conflict related to the present subject matter or to any materials discussed in this manuscript at any of our academic institutions or employers.

Open Access This article is distributed under the terms of the Creative Commons Attribution Noncommercial License which permits any noncommercial use, distribution, and reproduction in any medium, provided the original author(s) and source are credited.

\section{References}

1. Armstrong CG, Mow VC. Variations in the mechanical properties of human articular cartilage with age, degeneration, and water content. J Bone Joint Surg Am. 1982;64(1):88-94.

2. Nieminen MT, Töyräs J, Rieppo J, et al. Quantitative MR microscopy of enzymatically degraded articular cartilage. Magn Reson Med. 2000;43:676-81.

3. Lin PC, Reiter DA, Spencer RG. Classification of degraded cartilage through multiparametric MRI analysis. J Magn Reson. 2009;201:61-7.

4. Wayne JS, Kraft KA, Shields KJ, Yin C, Owen JR, Disler DG. MR imaging of normal and matrix-depleted cartilage: correlation with biomechanical function and biochemical composition. Radiology. 2003;228:493-9.

5. Liess C, Lüsse S, Karger N, Heller M, Glüer CC. Detection of changes in cartilage water content using MRI T2-mapping in vivo. Osteoarthr Cartil. 2002;10:907-13.

6. Nishii T, Kuroda K, Matsuoka Y, Sahara T, Yoshikawa H. Change in knee cartilage T2 in response to mechanical loading. J Magn Reson Imaging. 2008;28(1):175-80.

7. Li X, Ma CB, Link TM, et al. In vivo $\mathrm{T} 1 \rho$ and $\mathrm{T} 2$ mapping of articular cartilage in osteoarthritis of the knee using 3 T MRI. Osteoarthr Cartil. 2007;15(7):789-97.

8. Duvvuri U, Kudchodkar S, Reddy R, Leigh JS. T1 $\rho$ relaxation can assess longitudinal proteoglycan loss from articular cartilage in vitro. Osteoarthr Cartil. 2002;10:838-44.
9. Mlynárik V, Trattnig S, Huber M, Zembsch A, Imhof H. The role of relaxation times in monitoring proteoglycan depletion in articular cartilage. J Magn Reson Imaging. 1999;10:497-502.

10. Nissi MJ, Rieppo J, Töyräs J, et al. Estimation of mechanical properties of articular cartilage with MRI-dGEMRIC, T2 and T1 imaging in different species with variable stages of maturation. Osteoarthr Cartil. 2007;15:1141-8.

11. Mlynárik V, Sulzbacher I, Bittsanský M, Fuiko R, Trattnig S. Investigation of apparent diffusion constant as an indicator of early degenerative disease in articular cartilage. J Magn Reson Imaging. 2003;17(4):440-4.

12. Wheaton A, Casey FL, Gougoutas AJ, et al. Correlation of T1 $\rho$ with fixed charge density in cartilage. J Magn Reson Imaging. 2004;20:519-25.

13. Knauss R, Schiller J, Fleischer G, Kärger J, Arnold K. Self-diffusion of water in cartilage and cartilage components as studied by pulsed field gradient NMR. Magn Reson Med. 1999;41:285-92.

14. Burstein D, Gray ML, Hartman AL, Gipe R, Foy BD. Diffusion of small solutes in cartilage as measured by nuclear magnetic resonance (NMR) spectroscopy and imaging. J Orthop Res. 1993;11(4):46578.

15. Ateshian GA. The role of interstitial fluid pressurization in articular cartilage lubrication. J Biomech. 2009;42:1163-76.

16. Juras V, Bittsansky M, Majdisova Z, et al. In vitro determination of biomechanical properties of human articular cartilage in osteoarthritis using multi-parametric MRI. J Magn Reson. 2009;197 (1):40-7.

17. Fragonas E, Mlynarik V, Jellus V, et al. Correlation between biochemical composition and magnetic resonance appearance of articular cartilage. Osteoarthr Cartil. 1998;6:24-32.

18. Goodwin DW, Wadghiri YZ, Zhu H, Vinton CJ, Smith ED, Dunn JF. Macroscopic structure of articular cartilage of the tibial plateau: influence of a characteristic matrix architecture on MRI appearance. Am J Roentgenol. 2004;182:311-8.

19. Smith HE, Mosher TJ, Dardzinski BJ, et al. Spatial variation in cartilage T2 of the knee. J Magn Reson Imaging. 2001;14:50-5.

20. Niinimäki J, Korkiakoski A, Ojala O, Karppinen J, Ruohonen J, Haapea M, et al. Association between visual degeneration of intervertebral discs and the apparent diffusion coefficient. Magn Reson Imaging. 2009;27(5):641-7.

21. Sakai N, Hagihara Y, Furusawa T, Hosoda N, Sawae Y, Murakami T: Analysis of biphasic lubrication of articular cartilage loaded by cylindrical indenter. Tribology International In Press, Corrected Proof. 2011;1-12.>

22. Park S, Krishnan R, Nicoll SB, Ateshian GA. Cartilage interstitial fluid load support in unconfined compression. J Biomech. 2003;36:1785-96.

23. Li LP, Korhonen RK, Iivarinen J, Jurvelin JS, Herzog W. Fluid pressure driven fibril reinforcement in creep and relaxation tests of articular cartilage. Med Eng Phys. 2008;30(2):182-9.

24. Li LP, Herzog W, Korhonen RK, Jurvelin JS. The role of viscoelasticity of collagen fibers in articular cartilage: axial tension versus compression. Med Eng Phys. 2005;27(1):51-7.

25. Lai WM, Mow VC. Biphasic indentation of articular cartilage-I. Theoretical analysis. J Biomech. 1987;20:703-14.

26. Greene GW, Zappone B, Zhao B, et al. Changes in pore morphology and fluid transport in compressed articular cartilage and the implications for joint lubrication. Biomaterials. 2008;29(33):4455-62.

27. Ohmori Y, Tsukamoto Y. A study on the volume and chemistry of fluid exuded from normal articular cartilage under pressure. Kitasato Med. 1991;21:288-97.

28. Timonen MA, Töyräs J, Aula AS, Karjalainen JP, Riekkinen O, Jurvelin JS. Technical and practical improvements in arthroscopic indentation technique for diagnostics of articular cartilage softening. J Med Eng Technol. 2011;35(1):40-6. 